\title{
September 2017 Imaging Case of the Month
}

\author{
Michael B. Gotway, MD \\ Department of Radiology \\ Mayo Clinic Arizona \\ Scottsdale, Arizona USA
}

Clinical History: A 48-year-old woman with no previous medical history presented with complaints of intermittent cough persisting several months following a recent upper respiratory tract infection. No hemoptysis was noted.

Physical examination was largely unremarkable and the patient's oxygen saturation was $98 \%$ on room air. Upon close inspection, the right thorax appeared slightly asymmetrically smaller than the left.

Laboratory evaluation was unremarkable. Quantiferon testing for Mycobacterium tuberculosis was negative, and testing for coccidioidomycosis was unrevealing. Frontal and lateral chest radiography (Figure 1) was performed.

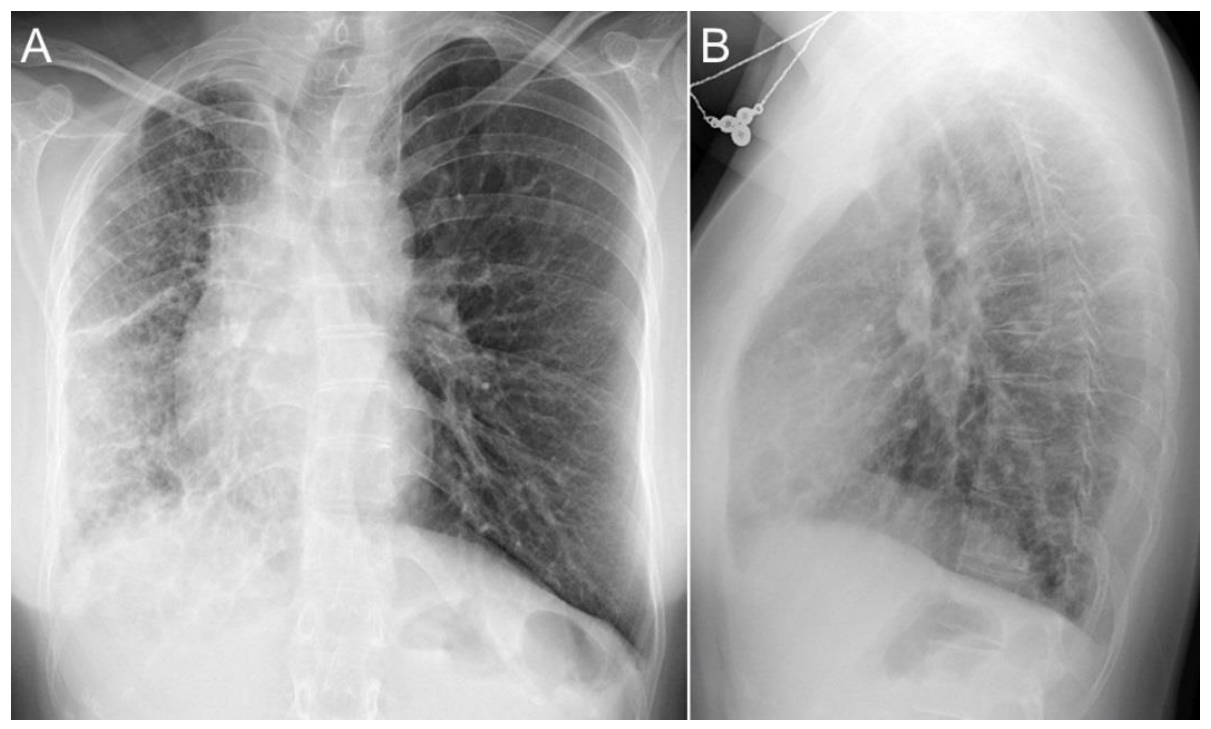

Figure 1. Frontal $(A)$ and lateral $(B)$ chest radiography

Which of the following statements regarding the chest radiograph is most accurate?

1. The chest radiograph shows asymmetric reticulation and interlobular septal thickening

2. The chest radiograph shows bilateral reticulation associated with decreased lung volumes

3. The chest radiograph shows large lung volumes

4. The chest radiograph shows multifocal consolidation and pleural effusion

5. The chest radiograph shows small cavitary pulmonary nodules 


\section{Correct! \\ 1. The chest radiograph shows asymmetric reticulation and interlobular septal thickening}

The frontal chest radiograph shows unilateral (right-sided) linear and reticular opacities, associated with decreased volume affecting the right thorax. There is no reticulation, septal thickening, or volume loss on the left side. No nodules, cavitary or otherwise, are present. There is no evidence of consolidation. The right lung volume is diminished, whereas the left lung volume is normal; the lung volumes are not abnormally large. No clear pleural effusion is present, although some thickening of the right-sided fissures, versus subpleural edema, is present.

Which of the following is not an appropriate differential diagnostic consideration for the chest radiographic pattern present?

1. Congenital unilateral pulmonary venous atresia

2. Interruption of the right pulmonary artery

3. Previous surgical resection

4. Pulmonary agenesis

5. Pulmonary hypoplasia 


\section{Correct! \\ 4. Pulmonary agenesis}

Interruption of the pulmonary artery (in this case, right-sided, which is the most common form of this rare anomaly), congenital unilateral pulmonary venous atresia, and pulmonary hypoplasia are developmental abnormalities of the thorax which may be associated with volume loss on the affected side of the thorax. Previous surgical resection can also produce unilateral thoracic volume loss. Other conditions that may produce unilateral thoracic volume loss include endobronchial obstruction and visceral pleural restriction due to post-inflammatory pleural thickening or neoplasia. Pulmonary agenesis (and pulmonary aplasia; see July 2017 Case of the Month) is also associated with significant unilateral volume loss, but, unlike the other entities listed, both pulmonary agenesis and aplasia are associated with both severe volume loss and opacity affecting the affected side of the thorax, due to complete shift of the heart, great vessels, and mediastinum into the affected thorax. The other conditions listed above are associated with unilateral volume loss, but because lung is present on the affected side, the affected thorax does not show increased opacity, unlike pulmonary agenesis and pulmonary aplasia.

Which of the following represents the most appropriate next step for the management of this patient?

1. ${ }^{133} \mathrm{Xe}$-Ventilation - ${ }^{99 m}$ Tc-perfusion scintigraphy

2. ${ }^{18}$ FDG-PET scan

3. Bronchoscopy

4. Contrast-enhanced thoracic CT

5. Contrast-enhanced thoracic MRI 


\section{Correct!}

\section{Contrast-enhanced thoracic CT}

Contrast-enhanced thoracic CT is the most appropriate modality for the investigation of the patient's chest radiographic abnormalities. Contrast-enhanced thoracic MRI could provide information similar to that obtainable with thoracic $\mathrm{CT}$, but the evaluation of the lung parenchyma and airways is far more efficacious with CT than MRI. ${ }^{18} \mathrm{FDG}-\mathrm{PET}$ scan is primarily used for lung cancer staging and the investigation of the indeterminate solitary pulmonary nodule and mediastinal abnormalities, and could prove useful subsequently, but is premature at this point. Similarly, bronchoscopy may prove valuable if an abnormality of the central lung, peribronchial regions, mediastinum, or airways is found, but is also premature at this point. ${ }^{133} \mathrm{Xe}$-Ventilation - ${ }^{99 m} \mathrm{Tc}$-perfusion scintigraphy could demonstrate the presence of either airway or vascular abnormalities contributing to the chest radiographic abnormalities, but often the findings at ventilation / perfusion scintigraphy are relatively non-specific and require interpretation in light of information from anatomic studies. The chest radiographic findings are not suggestive of thromboembolic disease, so ${ }^{133} \mathrm{Xe}$-Ventilation - ${ }^{99 m}$ Tc-perfusion scintigraphy's role in the evaluation of that disorder is not relevant for this patient.

The patient underwent contrast-enhanced thoracic CT for further investigation of the chest radiographic findings (Figure 2).

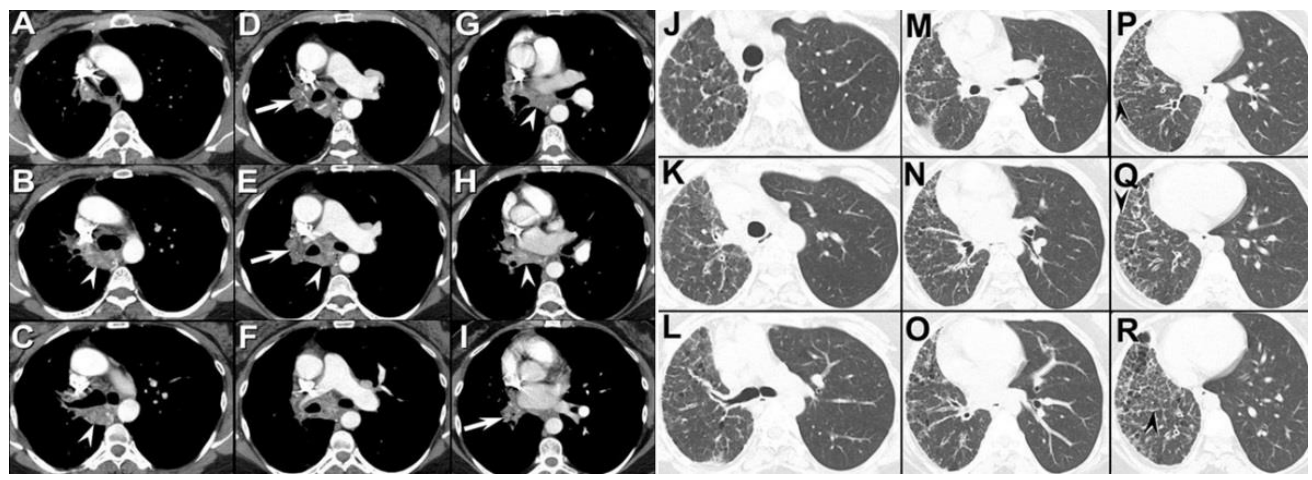

Figure 2. Representative images from the axial contrast-enhanced thoracic CT displayed in soft tissue windows (A-I) and lung windows (J-R).

Which of the following statements regarding this imaging study is most accurate?

1. The contrast-enhanced thoracic CT shows A multicystic space-occupying left lung abnormality

2. The contrast-enhanced thoracic CT shows abnormal right bronchial and mediastinal soft tissue

3. The contrast-enhanced thoracic CT shows active multifocal endobronchial obstruction

4. The contrast-enhanced thoracic CT shows dissection of the right pulmonary artery

5. The contrast-enhanced thoracic CT shows multifocal right pleural thickening 


\section{Correct! \\ 2. The contrast-enhanced thoracic CT shows abnormal right bronchial and mediastinal soft tissue}

The contrast-enhanced thoracic CT shows extensive left lung volume loss, but the volume loss is neither due to active endobronchial obstruction (the central airways bilaterally are patent) nor pleural thickening causing visceral pleural restriction (no pleural abnormality is seen on either side). Although the right pulmonary artery is clearly diminutive, no dissection or intrinsic abnormality of this vessel is seen. The left lung is entirely normal. The volume loss within the right thorax, evidenced by shift of the cardiomediastinal structures towards the right, is due to an intrinsic abnormality on that side, and is not the result of a space-occupying process, cystic or otherwise, on the left side. The contrast-enhanced thoracic CT does show abnormal soft tissue in the right peribronchial region and right mediastinum, extending into the subcarinal space and azygoesophageal recess and long the right aspect of the left atrium. The lung windows of this study show extensive reticulation and interlobular septal thickening through the hypoplastic-appearing right lung.

No prior imaging was available for the patient.

Which of the following represents the most appropriate next step for the management of this patient?

1. ${ }^{18}$ FDG-PET scan

2. Dynamic contrast-enhanced MRI

3. Bronchoscopy with endobronchial ultrasound

4. Video-assisted thoracoscopic lung biopsy

5. 1 or 3 


\section{Correct!}

\section{1 or 3}

Among the choices listed, the next step in management is a "close call"- both " 1 " and " 3 " are good choices. On the one hand, it could be argued that ${ }^{18}$ FDG-PET scan could be used to characterize the abnormal right peribronchial and mediastinal soft tissue and potentially detect additional sites of metabolically active abnormalities to target biopsy, particularly in light of the thoracic CT findings of abnormal right peribronchial and mediastinal soft tissue- perhaps the ${ }^{18} \mathrm{FDG}-\mathrm{PET}$ scan could disclose other more accessible abnormalities that would allow a diagnosis. On the other hand, it could be argued that the results of the ${ }^{18} \mathrm{FDG}-\mathrm{PET}$ scan are irrelevant- the abnormal soft tissue in the abnormal right peribronchial and mediastinal soft tissue has been well-shown and $\mathrm{CT}$ and requires a tissue diagnosis regardless of the ${ }^{18} \mathrm{FDG}$-PET scan findings. Videoassisted thoracoscopic tissue sampling would be capable of obtaining tissue for a definitive diagnosis, but it unnecessarily invasive at this point in the patient's evaluation. Dynamic contrast-enhanced thoracic MR (and CT) is a technique primarily used for characterization of indeterminate solitary pulmonary nodules and is not applicable to the patient.

At this point, which of the following is the least likely etiology for the patient's imaging findings?

1. Bronchogenic carcinoma

2. Fibrosing mediastinitis

3. Lymphangioleiomyomatosis

4. Metastatic malignancy

5. Sarcoidosis 


\section{Correct! \\ 3. Lymphangioleiomyomatosis}

Lymphangioleiomyomatosis is the least likely etiology for the patient's imaging findingsthe CT findings are largely non-cystic and are essentially unilateral in distribution, and both of these features are highly atypical for the diagnosis of lymphangioleiomyomatosis. The unilateral nature of the abnormalities on the imaging studies is also atypical for sarcoidosis, but the findings of interstitial and septal infiltration and abnormal peribronchial and mediastinal soft tissue are commonly seen in that disorder. The abnormal soft tissue in the peribronchial and mediastinal regions and the interlobular septal thickening are very common abnormalities at thoracic imaging studies in patients with fibrosing mediastinitis, metastatic malignancy, and bronchogenic malignancy.

The patient subsequently underwent ${ }^{18}$ FDG-PET scan (Figure 3).

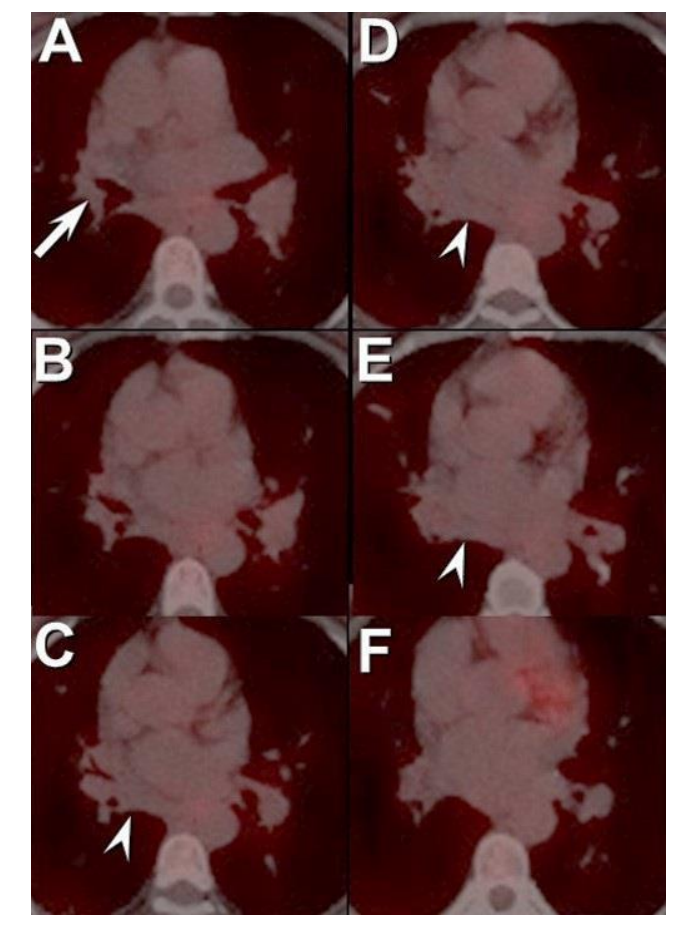

Figure 3. Axial 18FDG-PET scan.

Which of the following statements regarding this imaging study is most accurate?

1. The ${ }^{18}$ FDG-PET shows only focal tracer utilization with the abnormal mediastinal soft tissue

2. The ${ }^{18} \mathrm{FDG}-\mathrm{PET}$ shows only focal tracer utilization within the lung

3. The ${ }^{18} \mathrm{FDG}-\mathrm{PET}$ numerous foci of abnormal tracer uptake in the peribronchial regions, mediastinum, lung, and pleura

4. The ${ }^{18} \mathrm{FDG}-\mathrm{PET}$ shows no abnormal findings

5. The ${ }^{18}$ FDG-PET scan is technically limited 


\section{Correct! \\ 4. The 18FDG-PET shows no abnormal findings}

The ${ }^{18}$ FDG-PET scan is technically adequate and shows no tracer utilization at all- no foci of hypermetabolic tissue are identified within the thorax.

Which of the following represents the next most appropriate step for the evaluation of this patient?

1. Bronchoscopy with endobronchial ultrasound

2. Catheter pulmonary angiography

3. Endoscopic ultrasound

4. Pleuroscopy

5. Video-assisted thoracoscopic lung biopsy 


\section{Correct! \\ 1. Bronchoscopy with endobronchial ultrasound}

Given the lack of pleural abnormality, pleuroscopy would not be of benefit for this patient. Endoscopic ultrasound would be a useful procedure for evaluating the abnormal mediastinal soft tissue in this patient, but bronchoscopy with endobronchial ultrasound could not only address the mediastinal soft tissue but also sample the abnormal right peribronchial tissue, and transbronchial biopsy could be used to sample the infiltrative abnormality affecting the right lung interstitium, and therefore is the more useful procedure for this patient. Video-assisted thoracoscopic lung biopsy could also provide tissue for diagnosis, but is more invasive than bronchoscopy with endobronchial ultrasound and should be reserved if the latter procedure fails to provide a diagnosis. Catheter pulmonary angiography is most commonly employed as part of right heart catheterization for pulmonary hypertension evaluation or for the diagnosis and treatment of thromboembolic disease, but these disorders are not relevant to this patient.

The patient underwent bronchoscopy with endobronchial ultrasound-directed biopsy of the subcarinal soft tissue as well as transbronchial biopsy of the lung parenchyma- these tissue samples showed no specific abnormalities. The patient then subsequently underwent video-assisted thoracoscopic lung biopsy, which showed no evidence of malignancy or inflammatory change, either acute or chronic. Rather, regions of dilated anastomosing lymphatic channels associated with bundles of smooth muscle- more than simply lymphangiectasis- were noted and were felt to be consistent with a long-standing abnormality. No organisms were identified and no fibrosis was present. With this histopathological interpretation, the imaging findings were re-reviewed and it was noted that no pulmonary veins could be identified on the affected right side, and that this finding was likely developmental in nature. The abnormal peribronchial and mediastinal soft tissue has been described in congenital pulmonary venous atresia, and the hypoplastic right pulmonary artery and volume loss affecting the right thorax, in the absence of active airway obstruction, are more consistent with a developmental disorder than fibrosing mediastinitis. Finally, the complete lack of tracer utilization within the peribronchial and mediastinal soft tissue argues against both fibrosing mediastinitis and malignancy.

Diagnosis: Congenital unilateral pulmonary venous atresia

\section{References}

1. Ruano CA, Marinho-da-Silva A, Donato P. Congenital thoracic venous anomalies in adults: morphologic MR imaging. Curr Probl Diagn Radiol. 2015 Jul-Aug;44(4):337-

45. [CrossRef] [PubMed]

2. Rajiah P, Kanne JP. Computed tomography of pulmonary venous variants and anomalies. J Cardiovasc Comput Tomogr. 2010 May-Jun;4(3):155-63. [CrossRef] [PubMed]

3. Batram C, Strickland B. Pulmonary varices. Br J Radiol. 1971 Dec;44(528):927-35. [CrossRef] [PubMed] 
4. Tokunaga $\mathrm{K}$, Kubo T, Yamaoka T, Isoda H, Togashi K. Can the "pine-needle sign" on CT be used to differentiate pulmonary arteriovenous malformation from its mimics? Analysis based on dynamic contrast-enhanced chest CT in adults. Eur J Radiol 2017 (in press). [CrossRef]

5. Heyneman LE, Nolan RL, Harrison JK, McAdams HP. Congenital unilateral pulmonary vein atresia: radiologic findings in three adult patients. AJR Am J Roentgenol. 2001 Sep;177(3):681-5. [CrossRef] [PubMed] 